\title{
Variable Presentations of Benign Intra Cardiac Tumours - A Single Institution Study
}

\author{
Ajay Meena ${ }^{1}$, Budhaditya Chakraborty ${ }^{2}$ \\ ${ }^{1}$ Department of Cardiothoracic and Vascular Surgery, Mahatma Gandhi University of Medical Sciences \\ and Technology, Jaipur, Rajasthan, India. ${ }^{2}$ Department of Cardiothoracic and Vascular Surgery, \\ Mahatma Gandhi University of Medical Sciences and Technology, Jaipur, Rajasthan, India.
}

\section{ABSTRACT}

\section{BACKGROUND}

Benign cardiac tumours are a rare entity comprising of only about $0.2 \%$ of autopsy series.(1) In general, such tumours that present with a potential for embolization are to be dealt surgically as expeditiously as possible. However, there can be enormous variability in the presentations depending on the location and morphology of the tumour.

\section{METHODS}

We retrospectively reviewed our experience with benign intracardiac tumours over a period of 3 years at a single center. There were 10 patients, all of whom were managed surgically. Here we discuss the clinical presentations, operative management, operative outcomes, and follow up of this select group of patients. Intracardiac tumours constitute a rare entity of cardiac disease. The presentations of this pathology can be extremely variable. Sometimes detection maybe incidental on routine evaluation and at times they may present with the aftermath of systemic embolization, or rarely causing mechanical obstruction impacting cardiac output and function.

\section{RESULTS}

We present a spectrum of different benign intracardiac tumours and their variable presentations with their perioperative outcomes in a single institution over a span of three years. There were no perioperative deaths. Perioperative morbidity was restricted to one patient developing low cardiac output and severe mitral insufficiency.

\section{CONCLUSIONS}

The variability of their presentations and the different surgical approaches to handle each one of them represents to a large extent the entire pathophysiology of benign intracardiac tumours.

\section{KEY WORDS}

Tumours, Benign, Cardiac, Embolisation, Surgery
Corresponding Author: Dr. Budhaditya Chakroborty, Department of CTVS,

Mahatma Gandhi University of Medical

Sciences and Technology,

Sitapura, Jaipur-302022,

Rajasthan, India.

E-mail:drbrchakraborty@gmail.com

DOI: $10.14260 /$ jemds/2020/256

Financial or Other Competing Interests: None.

How to Cite This Article:

Meena A, Chakraborty B. Variable presentations of benign intra cardiac tumours- a single institution study. J. Evolution Med. Dent. Sci. 2020;9(14):11801183, DOI: $10.14260 /$ jemds/2020/256

Submission 18-12-2019,

Peer Review 15-03-2020,

Acceptance 21-03-2020,

Published 06-04-2020.

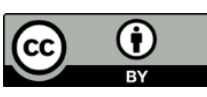




\section{BACKGROUND}

Primary cardiac tumours are a rare entity in majorly all surgical practices around the world. Secondary metastatic tumours constitute the largest number of these cases. However, $75 \%$ of these intracardiac tumours are benign. Presentations of these tumour masses can be extremely variable. Depending on their location and morphology the presentations maybe systemic [constitutional (fever, weight loss, fatigue) and paraneoplastic syndromes], embolic [pulmonary or systemic emboli], cardiac [mass effect causing valvular regurgitations, myocardial dysfunction, arrhythmias].

Diagnosis depends on a high index of suspicion and can almost always be made by echocardiography. Differentiation of cardiac tumours from valvular vegetation and atrial thrombus is important, and usually the echocardiographic appearance of a myxoma or a papillary fibroelastoma is quite distinctive. Benign tumours normally carry a good prognosis with normal life expectancy after resection. Patients who have had benign tumours resected are usually followed up with regular echocardiography and cardiology supervision. Malignant tumours such as sarcomas tend to have a poor outcome despite intervention, with a median survival from initial diagnosis of about 6 months. Occasional cases of survival due to complete resection do occur. Secondary malignancy affecting the heart has a grave outlook, although there is much that can be done to palliate the worst effects of the condition.

\section{METHODS}

A retrospective review of all the cases operated in the span of 3 years (2015-2018) at our institution were selected from our cardiac database and evaluated. We focused on the variability of clinical presentations, operative methods, perioperative challenges, histopathological studies, operative outcomes and follow up data. The follow up period ranged from 7 months to 28 months (mean 16 months).

\section{RESULTS}

A total of 10 patients were evaluated. About $70 \%$ of these tumours were LA myxomas (7 patients). 4 of these LA myxomas presented with vague constitutional symptoms of fatigue, low grade fever and episodes of palpitations. The rest 3 - one presented with features of CHF, one with history of stroke and one with history of acute aortobifemoral occlusion. Of the 7 patients, 5 were in atrial fibrillation. All LA myxomas were left sided, except one particular case where the large tumour invaded the IAS completely and partially bulged upon the right atrial cavity.

The remaining 3 patients were suspected cases of right ventricular hydatid cyst, RA myxoma and Right atrial tumour mass (thrombus, fibroelastoma). The patient with RV hydatid cyst was on investigation also detected to have an ectopic pregnancy and a hydatid cyst of the liver. All 3 patients with right sided tumours were in normal sinus rhythm.
Presentation for the right sided tumours were generally silent and picked up on incidental evaluations on echocardiography.

\section{Surgical Management}

All patients were subjected to open surgery under CPB. Aortobicaval cannulation with antegrade cardioplegic arrest was deployed. All LA Myxomas were approached trans septally. In one patient the myxoma was huge about $12 \times 10$ cms and abutted into the IAS and the pulmonary venous openings also bulging into the right atrial cavity. In all the cases the mitral valve patency was tested and degree of regurgitation, if mild on saline insufflation was accepted. A posterior Teflon felt annuloplasty was deployed in one case where the annulus was unusually enlarged with moderate to severe degree of regurgitation. For the RV tumour, the MPA was clamped and a right ventriculotomy was carried out. A classical hydatid cyst approx. $8 \times 6 \mathrm{cms}$ with active scolices was visualized. The fluid within the cyst was aspirated carefully after packing the surrounding tissues with sponges soaked in Povidone iodine. The Cyst capsule was dissected all around but found adherent firmly to the IVS. A small patch of the fibrous cyst wall was left adherent to the IVS in view of the risk of causing a VSR. The RV was closed using an autologous pericardial patch. The RA tumour mass was found embedded onto the IAS firmly. It was pedunculated, approximately $4 \times 2$ $\mathrm{cms}$ and had a filamentous attachment to the TV annulus near the ATL attachment. The entire Tumour mass with the IAS was excised and patched with autologous pericardium. The tricuspid valve specifically was unaffected by the tumour mass and competent on saline testing. All patients came off CPB uneventfully with minimal or no inotropic support. All tumours excised were sent for Histopathology examination.

There was no operative mortality in the above series. One patient of LA myxoma went into CHF in the post op period and the TTE revealed development of moderate - severe MR in spite of us having tested the mitral valve competence intra operatively by saline instillation. The case was medically managed and improved over a period of 8 days. The MR also significantly improved, and only residual trivial MR remained on follow up. HPE was confirmatory for all the myxomas. Histologically, all myxomas were characterized by the myxoma cell, a polygonal or stellate syncytial cell, forming cords or small nests. In a couple of tumours additional glandular and pseudocystic elements were found. The proteoglycan-rich myxoid, and vascularized stroma also contained dendritic cells, macrophages, and scattered lymphocytes. Haemorrhage and organizing thrombus resulted in abundant hemosiderin laden macrophages, which were present in all cases. One myxoma showed Gamma-Gandy bodies, elastic tissue, and calcification surrounding the hemosiderin. Degenerative changes included calcifications and ossifications.

The RV mass was confirmed to be Hydatid cyst. The cysts were surrounded by outer fibrous layer over the inner germinal layer and filled with clear hydatid fluid. The results re-confirmed that germinal layer is the primary site of parasite development. Echinococcal protoscolices were also noticed in some of the sections. There was slight haemorrhage, leucocyte infiltration and mild fibrous degeneration in the septal wall. Due to pressure effects from developing cysts, adjacent septal tissue showed atrophy, variable degeneration and lymphomononuclear infiltration. The smooth muscle adjacent to cysts 
was markedly congested and showed multiple small haemorrhagic areas.

The RA tumour mass was diagnosed as papillary fibroelastoma. Cardiac papillary fibroelastomas (CPFs) are the second most common primary cardiac tumours and the most common cardiac valvular tumours. Although they are histologically benign and usually asymptomatic, CPFs can lead to serious and life-threatening complications like myocardial infarction, stroke, pulmonary embolus, cardiac arrest etc. CPFs represent a rare entity in clinical medicine and literature regarding their management is limited. Histological section of the mass showed a benign papillary lesion comprised of a single layer of endocardial cells overlying a thin layer of mucopolysaccharide matrix and underlying an almost acellular, avascular stroma composed predominantly of elastic fibers.

\section{Follow Up}

All patients have been followed up on a regular basis. The duration of follow up ranged from 7 months to 28 months (mean 16 months). Follow up included symptomatology, clinical examination and echocardiographic follow up. One patient of LA myxoma who had moderate to severe Mitral regurgitation and a PA pressure of 52 (+ RA pressure) $\mathrm{mm}$ HG immediate post op showed regression in the degree of regurgitation over a period of 4 months. The PA pressures also diminished to 29 (+ RA pressure) $\mathrm{mm}$ HG on diuretics and afterload reduction. All patients irrespective of the tumour location were kept on diuretics for the first 3 months with concomitant beta blockers and ARBs for afterload reduction. All patients where the IAS and RV were patched were put on aspirin $150 \mathrm{mg}$ once daily for 3 months. There has been no recurrence of tumour mass in any of the operated patients on follow up as yet. The patient with right ventricular hydatid cyst was kept on Tab Albendazole for 6 months with monitoring of liver functions serially. She underwent a concomitant laparoscopic removal of liver hydatid cyst and removal of her ectopic pregnancy with sparing of her ovaries and fallopian tube in the same hospitalization and was discharged successfully. After a period of convalescence of 1 year she subsequently had an uneventful pregnancy and delivered a healthy female child through normal delivery.

\section{DISCUSSION}

WHO classification of tumours of the heart.

A. Benign Tumours and Tumour-like Lesions

- Rhabdomyoma.

- Histiocytoid cardiomyopathy.

- Hamartoma of mature cardiac myocytes.

- Adult cellular rhabdomyoma.

- Cardiac myxoma.

- Papillary fibroelastoma

- Haemangioma

- Cardiac fibroma.

- Inflammatory myofibroblastic tumour.

- Lipoma.

- Cystic tumour of the atrioventricular node.
B. Malignant Tumours

- Angiosarcoma

- Epithelioid haemangioendothelioma.

- Malignant pleomorphic fibrous histiocytoma.

- $(\mathrm{MFH}) /$ undifferentiated pleomorphic sarcoma.

- Fibrosarcoma and myxoid fibrosarcoma.

- Rhabdomyosarcoma.

- Leiomyosarcoma.

- Synovial sarcoma.

- Liposarcoma.

- Cardiac lymphomas.

- Metastatic tumours.

C. Pericardial Tumours

- Solitary fibrous tumour.

- Malignant mesothelioma.

- Germ cell tumours.

- Metastatic pericardial tumours.

\begin{tabular}{|c|c|c|}
\hline Sl. No. & Characteristic & Values [Mean \pm SD or $n(\%)]$ \\
\hline 1 & Age, years & $38+\_7$ \\
\hline 2 & $\begin{array}{l}\text { Gender } \\
\text { Male } \\
\text { Female }\end{array}$ & $\begin{array}{l}7 \\
3\end{array}$ \\
\hline & Median Duration of symptoms & 4 months \\
\hline 3 & $\begin{array}{c}\text { Symptomatology } \\
\text { Constitutional symptoms } \\
\text { CHF } \\
\text { Stroke } \\
\text { Vascular embolism }\end{array}$ & $\begin{array}{l}7 \\
1 \\
1 \\
1\end{array}$ \\
\hline 4 & $\begin{array}{c}\text { Rhythm } \\
\text { Sinus } \\
\text { Atrial fibrillation } \\
\text { Others/VPCs } \\
\end{array}$ & $\begin{array}{l}5 \\
5 \\
0\end{array}$ \\
\hline 5 & $\begin{array}{l}\text { LV function } \\
>50 \% \\
30-50 \% \\
<30 \% \\
\end{array}$ & $\begin{array}{c}8 \\
2 \\
\mathrm{Nil} \\
\end{array}$ \\
\hline 6 & $\begin{array}{l}\text { Tumour profile } \\
\text { LA Myxoma } \\
\text { RA Myxoma } \\
\text { RV hydatid cyst } \\
\text { RV fibroelastoma }\end{array}$ & $\begin{array}{c}10 \\
7 \\
1 \\
1 \\
1 \\
\end{array}$ \\
\hline 7 & Mean duration of follow up & 16 months(7 - 28 months) \\
\hline
\end{tabular}

Primary cardiac tumours are rare lesions with an autopsy incidence of $0.0017-0.03 \%$ including both benign and malignant histologic types.[1,2] The myxoma is the most frequent with an estimated incidence of $0.5 \%$ per million people per year.[3] Other benign tumours include papillary fibroelastomas, rhabdomyomas, fibromas, haemangiomas, lipomas and paragangliomas.[4,5] Amongst Myxomas, the left atrial myxomas remain the commonest while the right atrial or sometimes even biatrial myxomas are a rarer entity. We exclusively used a right atrial trans septal approach even for left sided tumours. The IAS was closed directly in some cases while an autologous pericardial patch was used for defect closure. The early operative outcomes are usually good with the biggest perioperative complication being stroke due to embolization. The risk of myxoma recurrence has been approximated to be $3 \%$ for sporadic tumours and $22 \%$ for familial myxomas. ${ }^{(6)}$

Amongst the right sided tumours, right ventricular hydatid cysts, Cardiac hydatidosis is rare (0.5-2\%) in comparison with hydatidosis of the liver (65\%) and lung (25\%). The right ventricle location is even rarer, $10 \%$ versus $60 \%$ for the left ventricle, and can cause fatal complications such as anaphylactic shock, dissemination, and pulmonary embolism. Several isolated case reports about RV hydatidosis have been 
published. The key in this surgery is to aspirate the potentially anaphylactic contents on the arrested heart and do an effective capitonnage. In our case, a small segment of the wall capsule attached to the IVS was left back. Fibroelastoma is another rare tumour usually attached to the valvular tissue - more of to the mitral and aortic valves. Tumours appeared round, oval, or irregular on echocardiography but were generally welldemarcated and homogenous in appearance. Most of them measure less than $20 \mathrm{~mm}$. Tricuspid fibroelastomas are particularly rare and there are few isolated case reports discussing them.

\section{CONCLUSIONS}

Benign cardiac tumours can have a wide array of clinical presentations. The histopathology of the tumour mass determines the outcomes in the immediate and long-term period. These tumours are usually operable with reasonably excellent surgical outcomes. Histopathologic study of the excised tumour mass is mandatory and helps determine the future treatment course.

\section{REFERENCES}

[1] Pacini D, Careddua L, Pantaleoa A, et al. Primary benign cardiac tumours: long-term results. European Journal of Cardio-Thoracic Surgery 2012;41:812-9.

[2] El Bardissi AW, Dearani JA, Daly RC, et al. Survival after resection of primary cardiac tumours:a 48-year experience. Circulation 2008;118(14 suppl 1):S7-S15.

[3] Steger CM, Hager T, Ruttmann E. Primary cardiac tumours: a single-center 41-year experience. Article ID 906109, ISRN Cardiology 2012;2012:1-7.

[4] L'aarje A, Lyazidi S, Kitane Y, et al. Cardiac hydatid cyst of the right ventricle: severe localization. Journal of Cardiology Cases 2017;16(4):138-40.

[5] Sun JP, Asher CR, Yang XS, et al. Clinical and echocardiographic characteristics of papillary fibroelastomas: a retrospective and prospective study in 162 patients. Circulation 2001;103(22):2687-93.

[6] Yandrapalli S, Mehta B, Mondal P, et al.Cardiac papillary fibroelastoma - the need for a timely diagnosis. World J Clin Cases 2017;5(1):9-13. 